\title{
Dynamic screening in solar plasma
}

\author{
Dan Mao \\ Department of Physics and Astronomy, University of Southern California, Los Angeles, \\ California, 90089, USA \\ Katie Mussack \\ Institute of Astronomy, University of Cambridge, Cambridge, CB3 OHA, UK \\ mussack@ast.cam.ac.uk \\ Werner Däppen \\ Department of Physics and Astronomy, University of Southern California, Los Angeles, \\ California, 90089, USA
}

\begin{abstract}
In the hot, dense plasma of solar and stellar interiors, Coulomb potentials are screened, resulting in increased nuclear reaction rates. Although Salpeter's approximation for static screening is widely accepted and used in stellar modeling, the question of screening in nuclear reactions has been revisited. In particular the issue of dynamic effects has been raised by Shaviv and Shaviv who apply the techniques of molecular dynamics to the conditions in the Sun's core in order to numerically determine the effect of screening. By directly calculating the motion of ions and electrons due to Coulomb interactions, the simulations are used to compute the effect of screening without the mean-field assumption inherent in Salpeter's approximation. In this paper we reproduce their numerical analysis of the screening energy in the plasma of the solar core and conclude that the effects of dynamic screening are relevant and should be included when stellar nuclear reaction rates are computed.

Subject headings: Equation of state - nuclear reactions, nucleosynthesis, abundances - plasmas - Sun:general
\end{abstract}

\section{INTRODUCTION}

Under the extreme temperatures and densities of the solar core, the plasma is fully ionized. The free electrons and ions interact via the Coulomb potential

$$
U(r)=\frac{e^{2}}{r} .
$$


In this Coulomb system, nearby plasma is polarized by each ion. When two ions approach with the possibility of engaging in a nuclear reaction, each ion is surrounded by a screening cloud. Thus each ion is attracted by the electrons and repelled by the protons in its partner's cloud. The combined effect of the particles in the screening clouds on the potential energy of the pair of ions is referred to as screening. This screening effect reduces the standard Coulomb potential between approaching ions in a plasma to a screened potential which includes the contribution to the potential from the surrounding plasma. The reduced potential enables the ions to tunnel through the potential barrier more easily, thus enhancing fusion rates.

Salpeter (1954) derived an expression for the enhancement of nuclear reaction rates due to electron screening. By solving the Poisson-Boltzmann equation for electrons and ions in a plasma under the condition of weak screening $\left(\phi_{\text {interaction }}<<k_{B} T\right)$, Salpeter arrived at an expression for the screening energy that is equivalent to that of the Debye-Hückel theory of dilute solutions of electrolytes (Debye \& Hückel 1923),

$$
E_{\text {screen }}=\frac{e^{2}}{\lambda_{D}}
$$

where the Debye length, $\lambda_{D}$, is the characteristic screening length of a plasma at temperature $T$ with number density $n$,

$$
\lambda_{D}^{2}=\frac{\epsilon_{0} k_{B} T}{n e^{2}} .
$$

Although Salpeter's approximation for screening is widely accepted, several papers over the last few decades (e.g. Shaviv \& Shaviv 1996; Carraro et al. 1988; Weiss et al. 2001) have questioned either the derivation itself or the validity of applying the approximation to hot, dense, Coulomb systems like the plasma of the solar core. Various work deriving alternative formulae for electrostatic screening (Carraro et al. 1988; Opher \& Opher 2000; Shaviv \& Shaviv 1996; Shavchenko 1999; Lavagno \& Quarati 2000; Tsytovich 2000) were refuted in subsequent papers (see Bahcall et al. 2002, for a summary of arguments in Salpeter's defense). However, the question of dynamic screening remains open. Dynamic screening arises because the protons in a plasma are much slower than the electrons. They are therefore not able to rearrange themselves as quickly around individual faster moving ions. Since nuclear reactions require energies several times the average thermal energy, the ions that are able to engage in nuclear reactions in the Sun are such faster moving ions, which therefore may not be accompanied by their full screening cloud. Salpeter uses the mean-field approach in which the many-body interactions are reduced to an average interaction that simplifies calculations. This technique is quite useful in thermodynamical calculations that rely on the average behavior of the plasma. However, dynamic effects for the fast-moving, interacting ions lead to a screened potential that deviates from the average value. The nuclear reaction rates therefore differ from those computed with the mean-field approximation.

Shaviv \& Shaviv (1996, 1997, 2000, 2001) used the method of molecular dynamics to model 
the motion of charges in a plasma under solar conditions in order to investigate dynamic screening. The advantage of the molecular-dynamics method is that it does not assume a mean field. Nor does it assume a long-time average potential for the scattering of any two charges, which is necessary in the statistical way to solve Poisson's equation to obtain the mean potential in a plasma. Shaviv and Shaviv attribute the differences between their simulations and Salpeter's theory to dynamic effects. Since their claims have been met with skepticism, we have conducted independent moleculardynamics simulations to confirm the existence of dynamic effects.

\section{NUMERICAL METHODS}

For consistency, we developed molecular-dynamics simulations using the same basic numerical methods as Shaviv and Shaviv. To quell the skeptics, we evaluated the validity of each assumption and approximation before implementing it in our own simulations. Previous publications (Mao et al. 2004; Mao 2008; Mussack et al. 2006, 2007; Mussack 2007) describe this process and show that we did not find any errors in the methods used by Shaviv and Shaviv. Once we validated their techniques, we used our independently developed simulations to examine dynamic effects in screening.

Our numerical simulation consisted of a 3-dimensional box with 1000 particles (half protons and half electrons) interacting via the Coulomb potential. The temperature and density of the solar core $\left(T=1.6 \times 10^{7} \mathrm{~K}, \rho=1.6 \times 10^{5} \mathrm{~kg} / \mathrm{m}^{3}\right)$ provided the velocity distributions and inter-particle spacing. We applied periodic boundary conditions and the minimum-image convention. In order to deal with the long-range nature of the Coulomb potential, we implemented a cut-off radius. With this method, particles separated by a greater distance than the cut-off radius were not included in the potential sums as explained in section 2.1. Quantum effects were included through the use of effective potentials as desribed in section 2.2. Detailed analysis of the use of a cutoff radius and effective quantum potential as well as tests of our simulations can be found elsewhere (Mao et al. 2004; Mao 2008; Mussack et al. 2006, 2007; Mussack 2007).

\subsection{Dealing with Long-Range Forces}

The long-range nature of the Coulomb potential introduces additional challenges to a moleculardynamics simulation. For many of the materials commonly modeled using molecular dynamics, short-range potentials such as the Lennard-Jones potential of a simple fluid

$$
u(r)=4 \epsilon\left[\left(\frac{\sigma}{r}\right)^{12}-\left(\frac{\sigma}{r}\right)^{6}\right]
$$


are appropriate. This potential decreases drastically with the separation of the two particles. Therefore it can be truncated quite reasonably at a distance of $r_{\text {cut }}=(2.5-3) \sigma$. The Coulomb potential, on the other hand, decays slowly with particle separation. Particles at much greater distances must be included in order to accurately compute the force on each particle. Ideally, the contribution of each of the infinite image particles should be included in the potential sums and the potential should never be truncated. Unfortunately this would take infinite computing time and is therefore impractical. Fortunately, more efficient techniques have been developed to compute long-range interactions (see, for example, Frenkel \& Smit 2002; Allen \& Tildesley 1987).

In their simulations, Shaviv and Shaviv took advantage of the screening of the plasma to shorten the effective range of the potential. They assume that interactions between particles that are farther apart than a few Debye lengths will be screened by the surrounding plasma. We have tested the truncation of the potential and determined that this assumption is appropriate for our simulation (Mussack et al. 2007; Mussack 2007). This enables us to truncate the interactions beyond a few Debye lengths, reducing the infinte-range potential to a manageable long-range potential.

\subsection{Quantum Effects}

When particles in a two-component plasma are closer than the thermal deBroglie wavelength

$$
\Lambda_{\alpha}=\frac{h}{\sqrt{2 \pi m_{\alpha} k_{B} T}},
$$

the effects of quantum diffraction and symmetry become significant. The thermal deBroglie wavelengths for our system are shown in Table1.

The average interparticle distance in our model is $<r>=.203 a_{B}$. Since $\Lambda_{p} \ll<r>$, a classical treatment is generally sufficient for the protons. However, it will be common to have two electrons that are separated by a distance less than $\Lambda_{e}$, requiring quantum mechanical treatment. We therefore include the quantum approximations used by Shaviv \& Shaviv (1996), i.e. the effective pair potentials derived for a hydrogen plasma by Barker (1971); Deutsch (1977);

\begin{tabular}{ccc}
\hline & Mass & $\Lambda_{\alpha}$ \\
Particle & $\left(m_{e}\right)$ & $\left(a_{B}\right)$ \\
\hline \hline Proton & 1836 & .008488 \\
Electron & 1 & .3637 \\
\hline
\end{tabular}

Table 1: Thermal deBroglie Wavelenghts at $T=1.6 \times 10^{7} \mathrm{~K}$ 
Deutsch et al. (1978, 1979). Quantum diffraction effects are described by

$$
v_{\alpha, \beta}^{(d)}=\frac{e_{\alpha} e_{\beta}}{r}\left[1-\exp \left(\frac{-r}{\lambda_{\alpha, \beta}}\right)\right],
$$

where

$$
\lambda_{\alpha, \beta}=\frac{\hbar}{\sqrt{2 \pi \mu_{\alpha, \beta} k_{B} T}}
$$

and $\mu_{\alpha, \beta}$ is the reduced mass of the pair.

$$
\mu_{\alpha, \beta}=\frac{1}{\frac{1}{m_{\alpha}}+\frac{1}{m_{\beta}}}
$$

The exclusion principle between electrons is included by adding

$$
v_{e e}^{(s)}=k_{B} T \ln 2 \exp \left(\frac{-r^{2}}{\pi \hbar_{e e}^{2} \ln 2}\right) .
$$

These potentials only differ from the classical Coulomb potential at short distances, where quantum effects are relevant. Fig. 1 shows the contribution to the effective potential from quantum corrections to the electrons and protons. It is clear that quantum effects for electrons can not be neglected at the high temperature of the solar core. The correction for proton-proton pairs is negligible, but electron-proton pairs do experience diffraction effects at close distances. Therefore we include quantum corrections for both electron-electron and electron-proton interactions.

\subsection{Method}

The goal of this work is to compute the energy exchanged between a pair of approaching protons and the surrounding plasma in order to evaluate the screening energy and the dependence of the screening energy on the relative kinetic energy of the pair. In order to accomplish this goal, we use a method similar to that of Shaviv and Shaviv. The technique for tracking approaching proton pairs and the energy they exchange with the plasma is described below.

1. For each proton $i$, find all protons approaching it. Choose the closest approaching proton and call it the partner of proton $i$.

2. Track the pair as they approach and then move apart. Record their distance of closest approach, $r_{c}$, and the energy of the pair at their closest point

$$
E_{\text {pair }}^{c}=E_{\text {kinetic }}^{c}+\frac{e^{2}}{r_{c}} .
$$




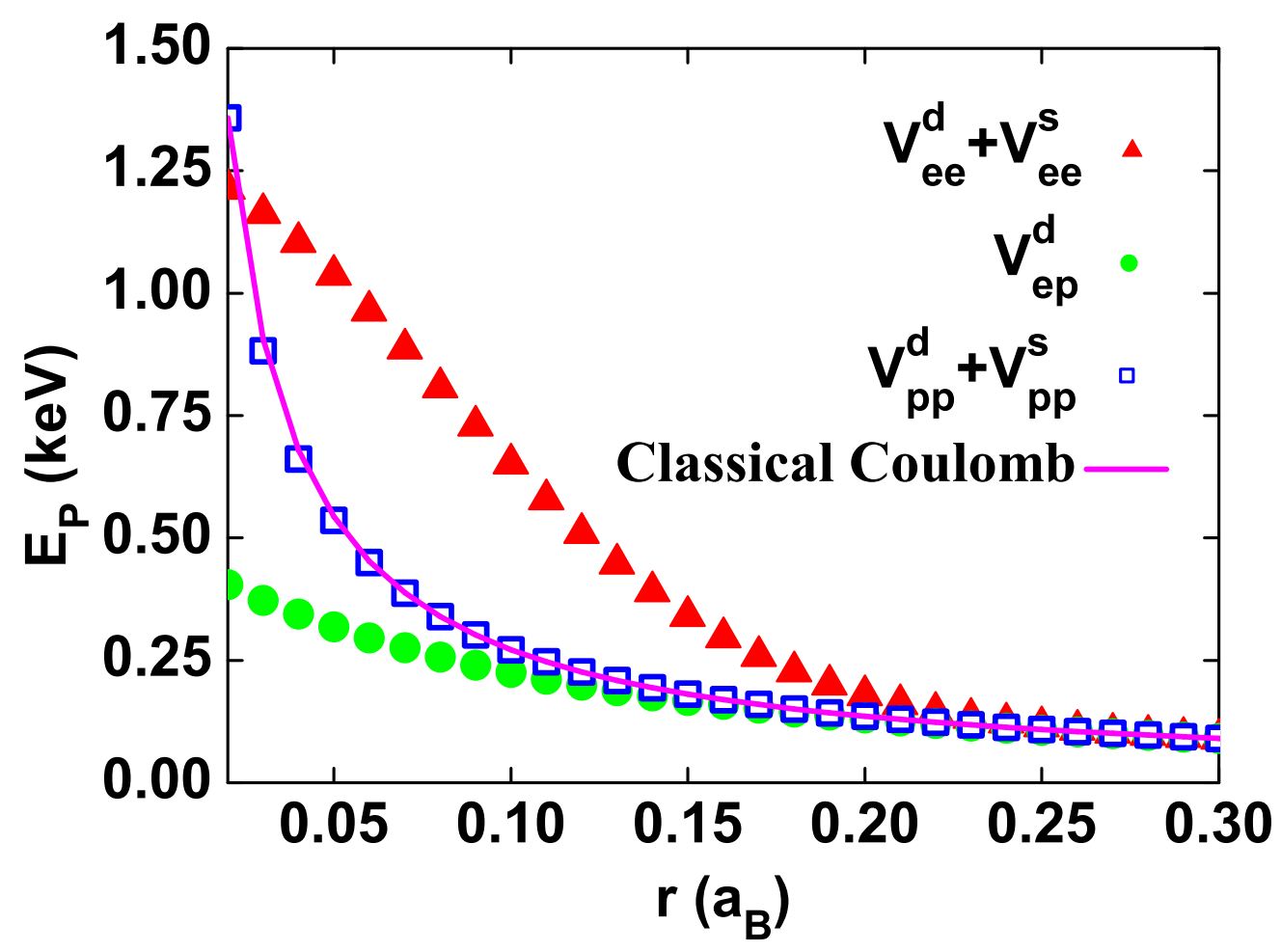

Fig. 1.- Comparison between effective pair potentials including quantum effects and the classical Coulomb potential (solid line) in a plasma under the conditions of the solar core. $V_{e e}$ (triangles) and $V_{p p}$ (squares) include both diffraction effects and symmetry effects, while $V_{e p}$ (circles) includes only diffraction effects. 
3. Continue tracking the pair until they are separated by a distance $R_{f}$. For our simulations, $R_{f}=2.0 a_{B}$ is a sufficient distance to represent the separation of the pair. At this point, record the far-apart energy of the pair

$$
E_{\text {pair }}^{f}=E_{\text {kinetic }}^{f}+\frac{e^{2}}{R_{f}} .
$$

Beyond this point, the pair is no longer tracked.

4. Find a new partner for proton $i$, and repeat the process for $i$ and its new partner.

Since a proton pair's departure from their closest point to a distance $R_{f}$ is symmetric to an approach from $R_{f}$ to $r_{c}$, we use the information obtained from the departure to examine the dynamic effect of the plasma on the screening enhancement for interacting proton pairs as they approach a separation at which nuclear reactions are possible. The dynamic information of each proton and its partner $d u$ jour are recorded at each time step.

\section{RESULTS}

First we examine the distribution of the distance of closest approach of proton pairs. Fig. 2 shows the number of pairs that achieved each closest distance $r_{c}$. We see that most pairs are able to get at least as close as the average distance of neighboring protons $\left\langle r_{p}>=0.256 a_{B}\right.$. As seen in Fig. 3, pairs of protons with higher far-apart kinetic energy $E_{\text {kinetic }}^{f}$ are more likely to get closer to each other than pairs with lower far-apart kinetic energy.

The screening energy is the energy exchanged between a pair of protons and the surrounding plasma. It represents the combined effect of the neighboring protons and electrons on the interacting pair. Salpeter's mean-field theory gives an expression for the average screened Coulomb potential that includes the energy exchanged between interacting protons and the plasma during their approach

$$
\overline{E_{\text {screen }}}(r)=\frac{e^{2}}{r}\left(1-e^{-r / \lambda_{D}}\right) \text {. }
$$

Salpeter's theory averages the effect of the kinetic energy of each particle so that the screening energy only depends on distance. However, our simulations show that the screening energy also depends on the kinetic energy of the protons. Protons with high kinetic energy tend to gain less energy from the plasma during the interaction, while protons with low kinetic energy gain more energy from the plasma. Shaviv \& Shaviv (2000, 2001) refer to this as the dynamic effect.

For the purpose of comparison, we define the screening energy in the simulations to be the difference in the energy of the pair when they are far apart and when they are close together.

$$
E_{\text {screen }} \equiv E_{\text {pair }}^{c}-E_{\text {pair }}^{f}
$$




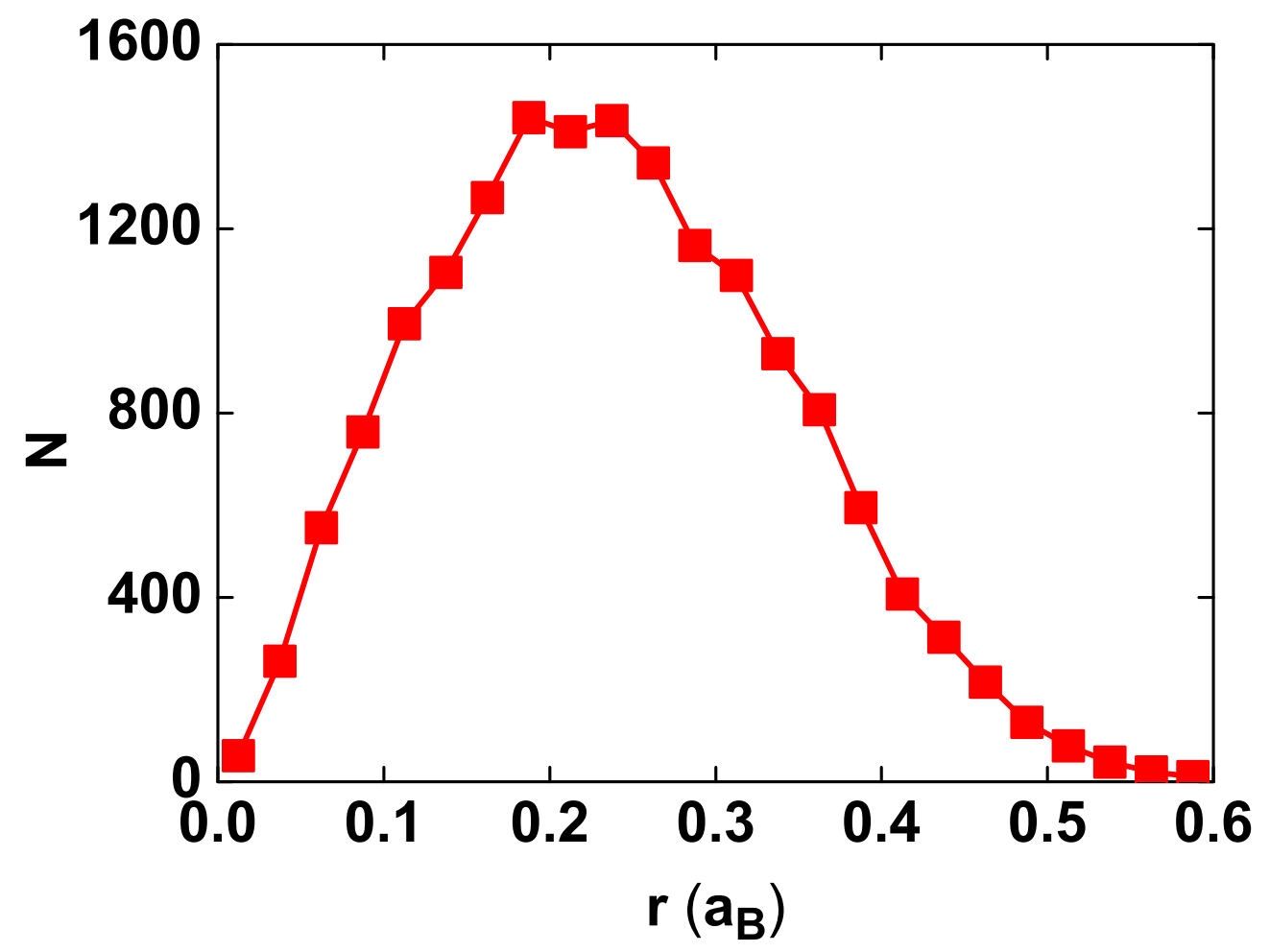

Fig. 2.- Distribution of the closest distance $r_{c}$. 


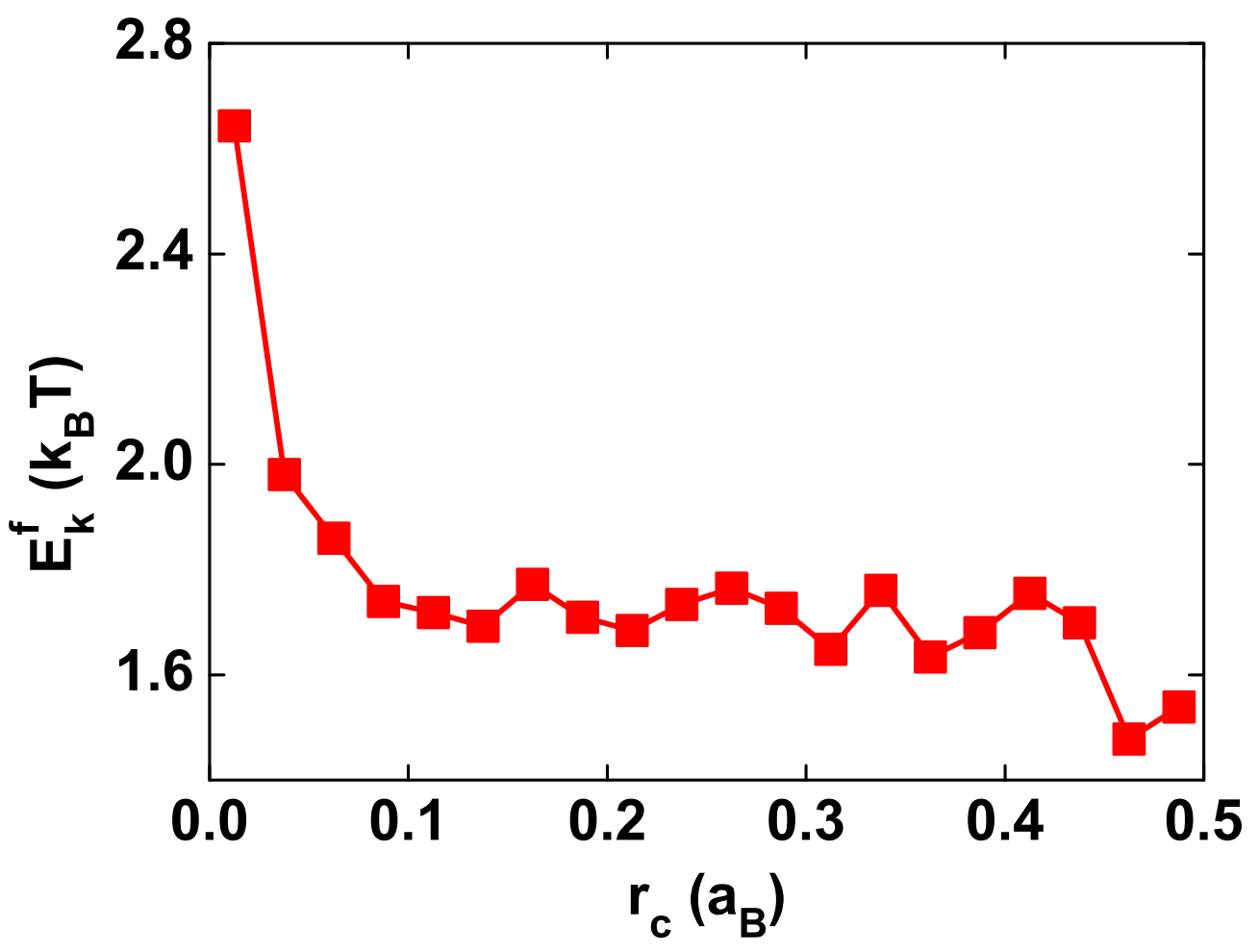

Fig. 3.- The relation between the average kinetic energy of proton pairs when they are far apart $E_{\text {kinetic }}^{f}$ and the closest separation of the pair $r_{c}$. 


$$
\begin{gathered}
E_{\text {pair }}^{c}=E_{\text {kinetic }}^{c}+\frac{e^{2}}{r_{c}} \\
E_{\text {pair }}^{f}=E_{\text {kinetic }}^{f}+\frac{e^{2}}{R_{f}} .
\end{gathered}
$$

Since all pairs have the same far-apart distance $R_{f}$,

$$
E_{\text {potential }}^{f}=\frac{e^{2}}{R_{f}}=\text { constant. }
$$

This yields a screening energy of

$$
E_{\text {screen }}=E_{\text {kinetic }}^{c}-E_{\text {kinetic }}^{f}+\frac{e^{2}}{r_{c}}-E_{\text {potential }}^{f} .
$$

A key ingredient in this equation for the screening energy is the difference in the kinetic energy of a pair when the partner protons are far apart and when they are at their closest separation.

$$
\Delta E_{\text {kinetic }}=\left(E_{\text {kinetic }}^{f}-E_{\text {kinetic }}^{c}\right)
$$

Here we examine the relationship between the average change in the kinetic energy of a pair and their distance of closest approach, shown in Fig. 4. This energy change is compared with the Coulomb potential and the screened Coulomb potential from Debye-Hückel theory. We see that at the distance of closest approach, the average kinetic energy exchanged between a pair of protons and the surrounding plasma has the form of the screened Coulomb potential.

$$
<\Delta E_{\text {kinetic }}>\left.\right|_{r_{c}} \sim \frac{e^{2}}{r_{c}} e^{-r_{c} / \lambda_{D}}
$$

On average, the protons interact in a mean field potential of

$$
V(r)=\frac{e^{2}}{r} e^{-r / \lambda_{D}}
$$

as Salpeter's theory states.

We can rewrite the average screening energy as

$$
<E_{\text {screen }}>\left.\right|_{r_{c}} \sim \frac{e^{2}}{r_{c}}\left(1-e^{-r_{c} / \lambda_{D}}\right)-E_{\text {potential }}^{f} .
$$

As $R_{f} \rightarrow \infty, E_{\text {potential }}^{f} \rightarrow 0$, leaving us with

$$
<E_{\text {screen }}>\left.\right|_{r_{c}}=\overline{E_{\text {screen }}}\left(r_{c}\right) .
$$




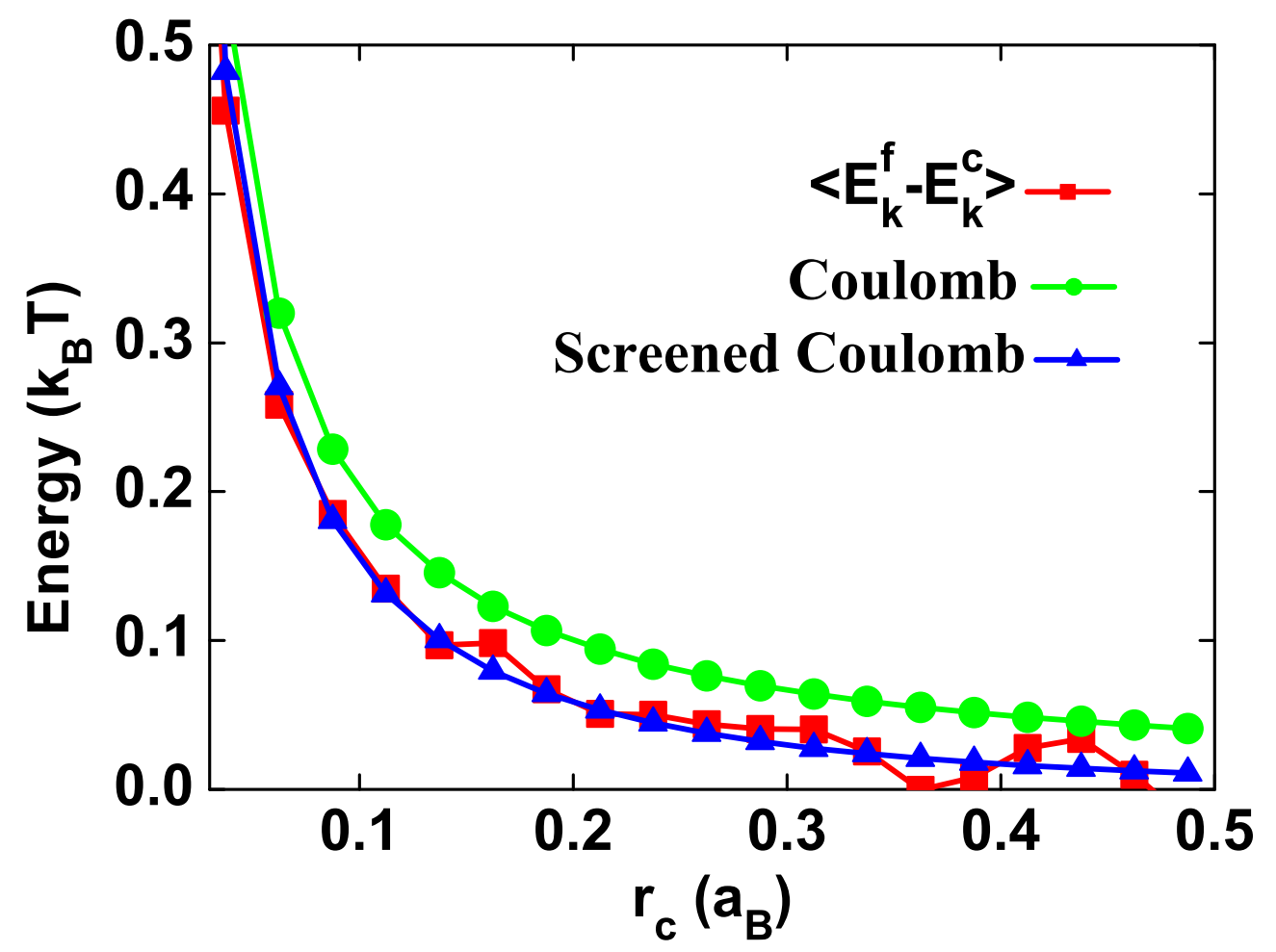

Fig. 4.- Dependence of the average kinetic energy change $<\Delta E_{\text {kinetic }}>$ on the closest distance $r_{c}$, compared with the Coulomb potential and screened Coulomb potential. 
The average screening energy in the simulation is the same as the average screening energy derived in Salpeter's theory. This confirms that on average, the system can be described by the screened Coulomb potential of the Debye-Hückel theory as Salpeter contends.

In order to see the dynamic effect on screening, we must look at the relationship between the screening energy and the far-apart kinetic energy of proton pairs. Fig. 5 shows the average energy gained from the plasma by pairs of protons with a given far-apart kinetic energy in the simulation. For comparison, the Debye-Hückel screening energy computed at a distance equal to the average closest-approach disance of pairs of protons with each far-apart kinetic energy is also shown. The screening energy of a pair of interacting protons depends on the relative kinetic energy of the pair. Pairs with far-apart kinetic energy greater than the thermal energy tend to gain less energy from the plasma than the mean-field screening energy, while pairs with lower far-apart kinetic energy gain energy more energy from the plasma than the mean-field screening energy.

$$
\begin{aligned}
& E_{\text {screen }}>\overline{E_{\text {screen }}} \text { for } E_{\text {kinetic }}^{f}<k_{B} T \\
& E_{\text {screen }}<\overline{E_{\text {screen }}} \text { for } E_{\text {kinetic }}^{f}>k_{B} T
\end{aligned}
$$

When the screening energy from the simulation is averaged over pairs with all $E_{\text {kinetic }}^{f}$, the result is the same as in Salpeter's theory. This reaffirms that Salpeter's theory is appropriate as a mean-field treatment.

However, the dynamic effect becomes relevant when pairs with high relative kinetic energy are singled out, as in solar nuclear reactions. Pairs with far-apart kinetic energy near the Gamov energy of $\mathrm{p}+\mathrm{p}$ reactions $\left(4.8 k_{B} T\right)$ will, on average, lose energy to the plasma rather than gaining energy. Because of this dependence of the screening energy on the relative kinetic energy of a pair of interacting particles, we conclude that the mean-field approach is not appropriate for computing nuclear reaction rates.

\section{CONCLUSIONS}

Our simulations confirm that the static Debye screening potential describes the average behavior of the plasma as a whole. Therefore it remains the appropriate treatment for mean-field properties of the system. However, dynamic effects are important when particles are singled out based on their velocities. Pairs of particles with greater relative velocities experience less screening than pairs of particles with lower relative velocities. This effect is relevant in nuclear reactions in

the Sun because they occur at energies that are several times greater than the thermal energy. The fast protons from the high-end tail of the Maxwell-Boltzmann velocity distribution are most likely 


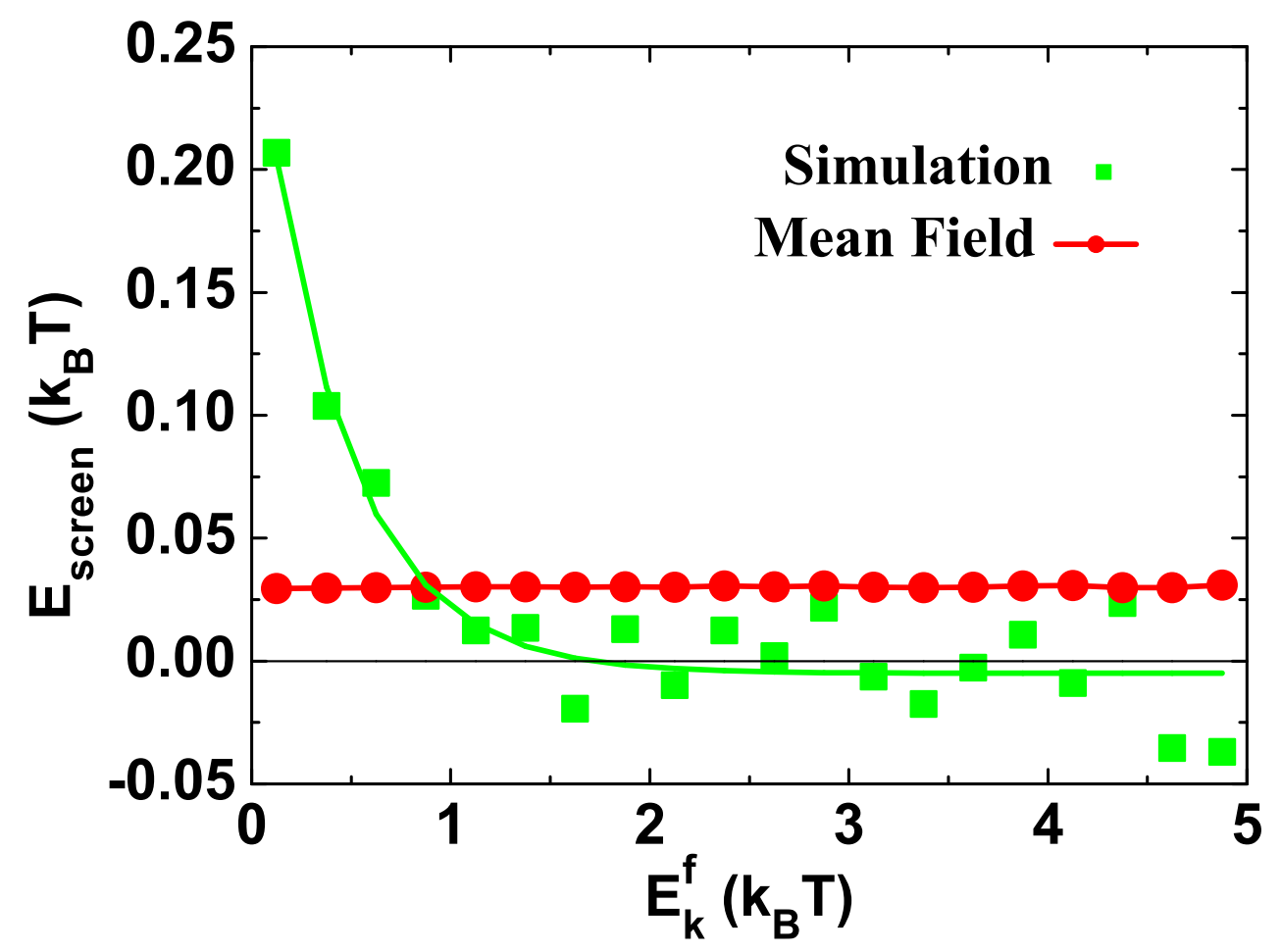

Fig. 5.- Dependence of the screening energy from the simulation $E_{\text {screen }}$ (squares) on the farapart kinetic energy $E_{\text {kinetic }}^{f}$. The Debye-Hückel screening energy computed at the averege closestapproach distance of pairs of protons with a given far-apart kinetic energy is shown (circles) for comparison. 
to take part in these reactions. Thus the mean-field approach is not valid in this case. We agree with Shaviv and Shaviv that the formalism for screening in solar nuclear reaction rates should be amended to account for this dynamic effect.

We thank Rajiv Kalia, Aiichiro Nakano, and Priya Vashishta for their guidance on the molecular-

dynamics simulations. This work was supported by grant AST-0708568 of the National Science Foundation.

\section{REFERENCES}

Allen, M.P. and Tildesley, D.J. 1987, Computer Simulation of Liquids (Oxford University Press)

Bahcall, J.N., Brown, L.S., Gruzinov, A., and Sawyer, R.F. 2002, AE्FA, 383, 291

Barker, A.A. 1971, J. Chem. Phys., 55,1751

Carraro, C., Schafer, A., and Koonin, S.E. 1988, ApJ, 331, 565

Debye, P. and Hückel, E. 1923, Z. Phys., 24,305

Deutsch, C. 1977, Phys. Lett., 60,317

Deutsch, C., Gombert, M.M. and Minoo, H. 1978, Phys. Lett., 66A,381

Deutsch, C., Gombert, M.M. and Minoo, H. 1979, Phys. Lett., 72A,481

Frenkel, D. and Smit, B. 2002, Understanding Molecular Simulation: from Algorithms to Applications (Academic Press)

Lavagno, A. and Quarati, P. 2000, Nucl. Phys. B (Proc. Suppl.),87,209

Mao, D., Mussack, K. and Däppen, W. 2004, in Proc. SOHO 14/GONG 2004 Workshop, ed. D. Danesy (ESA SP 559), 560

Mao, D., in progress, Proquest Dissertations And Theses.

Mussack, K., Mao, D. and Däppen, W. 2006, in Proc. SOHO 18/GONG 2006/HelAs I Workshop, ed. K. Fletcher, M. Thompson (ESA SP 624, 2006), 20.1

Mussack, K., Mao, D. and Däppen, W. 2007, in Proc. Unsolved Problems in Stellar Physics, AIP Conference Proceedings, vol. 948, ed. R. Stancliffe et al. (Melville, NY), 207 
Mussack, K. 2007, Proquest Dissertations And Theses 2007, 0208, 0606.

Opher, M., and Opher, R. 2000, ApJ, 535,473

Salpeter, E. E. 1954, Australian J. Phys., 7,373

Savchenko, V. I. 1999, Preprint (astro-ph/9904289).

Shaviv, N. J. and Shaviv, G. 1996, ApJ, 468,433

Shaviv, N. J. and Shaviv, G. 1997, ApESSS Lib., 214,43

Shaviv, G. and Shaviv, N. J. 2000, ApJ, 529,1054

Shaviv, N. J. and Shaviv, G. 2001, ApJ, 558,925

Tsytovich, V. N. 2000, AEA A, 356,L57

Weiss, A., Flaskamp, M., and Tsytovich, V. N. 2001, AEFA, 371,1123 